\title{
Integrated Global Optimization Model for Electrical Cables in Offshore Wind Farms
}

\author{
Pérez-Rúa, Juan-Andrés; Stolpe, Mathias; Cutululis, Nicolaos Antonio
}

Published in:

I E E E Transactions on Sustainable Energy

Link to article, DOI:

10.1109/TSTE.2019.2948118

Publication date:

2020

Document Version

Peer reviewed version

Link back to DTU Orbit

Citation $(A P A)$ :

Pérez-Rúa, J-A., Stolpe, M., \& Cutululis, N. A. (2020). Integrated Global Optimization Model for Electrical Cables in Offshore Wind Farms. I E E E Transactions on Sustainable Energy, 11(3), 1965-1974.

https://doi.org/10.1109/TSTE.2019.2948118

\section{General rights}

Copyright and moral rights for the publications made accessible in the public portal are retained by the authors and/or other copyright owners and it is a condition of accessing publications that users recognise and abide by the legal requirements associated with these rights.

- Users may download and print one copy of any publication from the public portal for the purpose of private study or research.

- You may not further distribute the material or use it for any profit-making activity or commercial gain

- You may freely distribute the URL identifying the publication in the public portal 


\title{
Integrated Global Optimization Model for Electrical Cables in Offshore Wind Farms
}

\author{
Juan-Andrés Pérez-Rúa, Mathias Stolpe, and Nicolaos A. Cutululis, Senior Member, IEEE
}

\begin{abstract}
A MILP program for integrated global optimization of electrical cables systems in Offshore Wind Farms (OWFs) is presented. Electrical cables encompass the cable layout in collection systems to interconnect Wind Turbines (WTs), and transmission systems to couple Offshore Substations (OSSs) to the Onshore Connection Point (OCP). The program is solved through a modern branch-and-cut solver, demonstrating the ability to tackle large-scale instances with hundreds of WTs and several OSSs. The model supports as objective function the initial investment plus economic losses due to total electrical power losses. The importance and functionality of incorporating electrical losses is demonstrated, along with the need to simultaneously optimize the cable layout, OSSs location, and transmission cables. The method is tested for three case studies. The results show that (i) points near the global optimum, with an imposed maximum tolerance, are calculable within reasonable computational time and effort, and (ii) the integrated model can be much more efficient than a benchmark approach based on enumeration, i.e., exhaustive evaluation of all possible optimization problems derived from unique OSSs locations.
\end{abstract}

Index Terms-Offshore wind energy, Cable layout, Global optimization, Mixed integer linear programming, Medium voltage cables, High voltage cables.

\section{NOMENCLATURE}

\section{Acronyms}

OWF(s) Offshore Wind Farm(s).

WT(s) Wind Turbines(s).

OSS(s) Offshore Substation(s).

SCETM Single-Core Equivalent Thermal Model.

OCP Onshore Connection Point.

L Length.

LP Length plus total Power losses.

I Initial investment.

IL Investment plus total Power losses.

SY Synthetic.

SYs Single synthetic.

LA London Array.

\section{Parameters (non-sets)}

$\begin{array}{ll}n_{w} & \text { Number of wind turbines. } \\ n_{o} & \text { Number of required offshore substations. } \\ \beta_{t} & \begin{array}{l}\text { Number of potential locations for offshore subs- } \\ \text { tations. }\end{array} \\ d_{i j} & \text { Euclidean norm for arc }(i, j) . \\ u_{c_{t}} & \text { Capacity of cable } t \text { for collection system in num- }\end{array}$

Juan-Andrés Pérez-Rúa, Mathias Stolpe, and Nicolaos A. Cutululis are with the Department of Wind Energy, Technical University of Denmark, Frederiksborgvej 399, 4000 Roskilde, Denmark (e-mail: juru@dtu.dk)

Manuscript received XXX, XXX; revised XXX, XXX. ber of wind turbines.

$u_{e_{t}} \quad$ Capacity of cable $t$ for transmission system in number of wind turbines.

$c_{c_{t}} \quad$ Metric initial investment cost of cable $t$ for collection system.

$c_{e_{t, n}} \quad$ Metric initial investment cost of cable $t$ for transmission system, with $n$ cables per phase.

$I_{c_{t}} \quad$ Steady-state rated current of cable $t$ for collection system.

$I_{e_{t}} \quad$ Steady-state rated current of cable $t$ for transmission system.

$V_{n_{c}} \quad$ Nominal line-to-line voltage of the collection system.

$V_{n_{e}} \quad$ Nominal line-to-line voltage of the transmission system.

$P_{n} \quad$ Nominal power of the wind turbines.

$S_{r c_{t}} \quad$ Nominal power of cable $t$ for collection system.

$S_{r e_{t}} \quad$ Nominal power of cable $t$ for transmission systems.

$\overrightarrow{\gamma_{t}} \quad$ Propagation constant of cable $t$.

$\overrightarrow{Z_{c_{t}}} \quad$ Characteristic impedance of cable $t$.

$\overrightarrow{z_{t}}$

$\overrightarrow{y_{t}}$

$I_{c_{i j, t}}^{\vec{k}}$ Metric series impedance of cable $t$.

Metric admittance of cable $t$.

Nominal phasor current of arc $(i, j)$ using cable $t$, for collection system, when $k$ wind turbines are connected.

$I_{e_{i j, t, n}}^{\vec{k}} \quad$ Nominal phasor current of arc $(i, j)$ using cable $t$, for transmission system, when $k$ wind turbines are connected, with $n$ cables per phase.

$S_{c_{i j, t}}^{k} \quad$ Nominal power of arc $(i, j)$ using cable $t$, for collection system, when $k$ wind turbines are connected.

$p^{\omega} \quad$ Power produced by a wind turbine at $\omega$ hour-slot.

$f_{i j}^{\omega, k} \quad$ Power flow in arc $(i, j)$ at $\omega$ hour-slot,

$I_{c_{i j, t}}^{\overrightarrow{\omega, k}}$ when $k$ wind turbines are connected.

Phasor current through arc $(i, j)$ using cable $t$, for collection system, at $\omega$ hour-slot, when $k$ wind turbines are connected.

$\lambda_{1} \quad$ Screen losses factor.

$\lambda_{2} \quad$ Armouring losses factor.

$W_{d_{t}} \quad$ Metric dielectric loss of cable $t$.

$R_{t} \quad$ Metric electrical resistance of cable $t$.

$l_{c_{i j, t}}^{\mu, k} \quad$ Annual total power losses through $\operatorname{arc}(i, j)$ using cable $t$, for collection system, at year $\mu$, when $k$ wind turbines are connected.

$l_{e_{i j, t, n}}^{\mu, k} \quad$ Annual total power losses through arc $(i, j)$ using cable $t$, for transmission system, at year $\mu$, when 
$k$ wind turbines are connected, with $n$ cables per phase.

$U_{c} \quad$ Capacity of the biggest cable available, for collection system, in number of wind turbines.

$r \quad$ Discount rate.

$c_{i j}^{k} \quad$ Metric cost of arc $(i, j)$, when $k$ wind turbines are connected.

$c_{l}^{o} \quad$ Independent term of linear cost function for offshore substation $l$.

$c_{l}^{l} \quad$ Dependent term of linear cost function for offshore substation $l$.

$\phi \quad$ Maximum number of feeders per offshore substation.

$\eta \quad$ Loading factor for offshore substations.

$v \quad$ Maximum number of wind turbines arcs set to a wind turbine.

$v_{f} \quad$ Maximum number of wind turbines arcs set to a wind turbine for feasibility problem.

$v_{f_{\min }} \quad$ Algorithm's parameters for feasibility problem.

$v_{f_{\delta}}, v_{f_{\max }}$

$v_{0} \quad$ Maximum number of wind turbines arcs set to a wind turbine for global optimization problem.

$v_{o_{\text {min }}} \quad$ Algorithm's parameters for global optim. problem. $v_{o_{\delta}}, v_{o_{\max }}$

$\epsilon \quad$ Required relative optimality gap in MILP solver.

\section{Parameters (sets)}

$\mathcal{N}_{o} \quad$ Set of sets of candidate locations for all offshore substations.

$\mathcal{N}_{\beta_{t}} \quad$ Set of candidate locations for all offshore substations.

$\mathcal{N}_{n_{o}} \quad$ Set of required offshore substations.

$\mathcal{N}_{o i} \quad$ Set of candidate locations for offshore substation $i$.

$\mathcal{N}_{w} \quad$ Set of wind turbines.

$\mathcal{N} \quad$ Set of offshore substations, wind turbines, and onshore connection point.

$G \quad$ Weighted directed graph.

$\mathcal{A} \quad$ Set of available arcs.

$\mathcal{D} \quad$ Set of arcs' weights.

$\mathcal{T}_{c} \quad$ Set of available cables for collection system.

$\mathcal{T}_{e} \quad$ Set of available cables for transmission system.

$\mathcal{U}_{c} \quad$ Set of cables' capacities for collection system in wind turbines number.

$\mathcal{U}_{e} \quad$ Set of cables' capacities for transmission system in wind turbines number.

$\mathcal{C}_{c} \quad$ Set of cables' initial investment costs for collection system.

$\mathcal{C}_{e} \quad$ Set of cables' initial investment costs for transmission system.

$\Omega^{\mu} \quad$ Set of hours-slot for a year $\mu$.

$\mathcal{M} \quad$ Set of operational years.

$G_{r} \quad$ First reduced graph.

$\mathcal{A}_{r} \quad$ Set of first reduced arcs.

$\mathcal{I} \quad$ Set of crossing pairs arcs.

$\mathcal{L} \quad$ Set of forbidden areas.

$\mathcal{A}_{\ell} \quad$ Set of arcs defining area $\ell$.
$\Upsilon_{i} \quad$ Set of wind turbines connected to $i$.

$G_{r}^{\prime} \quad$ Second reduced graph.

$\mathcal{A}_{\nabla}^{\prime} \quad$ Set of second reduced arcs.

$G_{r}^{\prime \prime} \quad$ Third reduced graph.

$\mathcal{A}_{\nabla}^{\prime \prime} \quad$ Set of third reduced arcs.

\section{Variables}

$x_{i j} \quad$ Binary variable to activate $\operatorname{arc}(i, j)$.

$y_{i j}^{k} \quad$ Binary variable to activate $\operatorname{arc}(i, j)$, when $k$ wind turbines are connected.

$z_{i, l} \quad$ Binary variable to choose candidate location $l$ for OSS $i$.

$x_{c_{i j, t}} \quad$ Binary variable for arc $(i, j)$ to select optimum cable type $t$, for collection system.

$x_{e_{i j, t, n}} \quad$ Binary variable for arc $(i, j)$ to select optimum cable type $t$, for transmission system, with $n$ cables per phase.

$\sigma_{i} \quad$ Integer variable modelling the number of wind turbines connected to offshore substation $i$.

$\sigma_{i, l} \quad$ Integer variable modelling the number of wind turbines connected to offshore substation $i$, at candidate location $l$.

\section{Subscripts}

$i \quad$ Point $\in \mathcal{N}$.

$j \quad$ Point $\in \mathcal{N}$.

$l \quad$ Point $\in \mathcal{N}_{o}$.

ij $\quad$ Arc $(i, j)$ with tail at $i$ and head at $j$.

$t \quad$ Cable type $\in \mathcal{T}_{c}$ or $\mathcal{T}_{e}$.

$n \quad$ Number of cables per phase.

$\ell \quad$ Forbidden area $\in \mathcal{L}$.

\section{Superscripts}

$k \quad$ Number of turbines connected in $(i, j)$.

$\omega \quad$ Hour-slot $\in \Omega^{\mu}$.

$\mu \quad$ Year $\in \mathcal{M}$.

\section{INTRODUCTION}

C OST reductions and operational performance enhancement are the main current challenges for designing and running Offshore Wind Farms (OWFs). Europe (mainly), Asia, and recently North America where the first large-scale installations are expected in 2023 [1], have contributed to the rapid proliferation of this technology for electrical power generation. The share of OWFs worldwide, with respect to the total installed power of wind energy ( $591 \mathrm{GW}$ ), is prognosed to increase from the actual $4 \%$ to more than $10 \%$ in 2025 , reaching a global installed capacity of of $100 \mathrm{GW}$.

Electrical power cables represent a crucial component of the Balance of Plants $(\mathrm{BoP})$ in OWFs, representing roughly $10 \%$ of the total capital expenses [2]. Between 2018 and 2028 more than $19,000 \mathrm{~km}$ of cables for collection systems are projected to be installed, with an estimated worth of $£ 5.36 \mathrm{bn}$ [3]. Likewise, required export cables with lengths in the order of hundreds of kilometers are the trend given the 
distancing of Offshore Substations (OSSs) to the Onshore Connection Point (OCP). Similarly, economies of scale pushes the development of large-scale OWFs, having more than 100 Wind Turbines (WTs), while increasing their rated power.

The importance of electrical cables in OWFs lies not only in the evident cost weight, but also in the operational performance of the whole project. Points of single failure due to electro-thermal and mechanical stress, and their mitigation, is one of the most studied topics in the field nowadays. Therefore, special attention must be addressed to designing systems involving electrical cables, such as the collection system, and the export system, while simultaneously accounting for the interrelation among them.

The integrated design of electrical cables in OWFs comprise the following aspects: (i) limited capacity of the cables' thermal rating, (ii) topological design of the cable layout, (iii) capital expense optimization in both the collection and the transmission systems, (iv) diminution of total electrical power losses, (v) clustering of WTs to OSSs, and (vi) geographical location of the OSSs. As demonstrated in [4], an instance of the main problem involving the points (i), (ii), and (iii) falls into the category of NP hard problems [5], mapping to a modified version of a Capacitated Minimum Spanning Tree (C-MST). By deduction, the main problem dealing simultaneously with all six aspects, inherit the complexity with additional design variables, turning this task into a very challenging subject in the context of computational optimization in OWFs.

In order to tackle the electrical cables design in OWFs, available methods can be classified as follows [6]. (i) Heuristics methods are all those algorithms that are bounded in computation time while developing a solution by construction in sequential steps [7]. (ii) Metaheuristics can be defined as improvement heuristics, which find fast initial feasible points (ideally), and by means of iterative processes, intend to improve them using different stochastic operators [8], [9]. (iii) Global optimization offers the enormous advantage to provide mathematical certifications for optimality in case of convex problems. However, for combinatorial problems, the required computational time is unknown and unpredictable, and for the range of modern large-scale OWFs, memory capacities start becoming an issue as well [10]. (iv) Combination of matheuristics, providing frameworks to cope with these challenges by limiting the search space, in combination with mathematical programs providing feasible points or the global optimum [4].

To the best of the authors' knowledge, only the following works have tackled the full integrated design of electrical cables for OWFs using global optimization. A MILP model combined with Benders decomposition is presented in [11], a MILP model [12] solved by branch-and-cut method, a MILP model with progressive contingence incorporation [13], and finally a MIQP model is proposed in [14].

The works [11], [13], [14] provide remarkable advances on stochastic optimization for problems in this context. Different stochastic scenarios are supported, accounting for wind power variability and cables failure. Distinctive theoretical strategies to accelerate convergence are applied and compared. While in [12] a single MILP model is formulated and later solved by branch-and-cut method. The model optimizes uniquely for initial investment, while also disregards important practical constraints, such as, cables crossings, and forbidden areas. Nevertheless, the application of those methods did not focus on large-scale OWFs with multiple OSSs, as the case studies were defined by real projects with maximum 30 WTs. State-of-the-art OWFs can encompass WTs in the order of hundreds.

Other works propose the use of metaheuristic for providing feasible points to tackle large-scale instances. This includes [15] where Particle Swarm Optimization is employed and, [16] in which a Genetic Algorithm is proposed. Given the intrinsic features of the used metaheuristics, there is no possibility to infer about the quality of the obtained solutions. In virtue of the above, in this manuscripts is proposed a MILP model embedded in an efficient algorithmic framework, able to compute global optimum solution points (or near to it) in reasonable computational time, for the full integrated design of electrical cables in large-scale OWFs. Additionally, the model quickly finds feasible points. Likewise, the proposed model supports a combined objective function defined by total system costs, i.e. initial investment and total electrical power losses of both the collection and transmission systems simultaneously.

Compared to [17], the authors propose the following generalizations: (i) modelling of transmission systems, (ii) a compact and single mathematical model which can be used to simultaneously optimize the collection and the transmission system, (iii) flexible locations of OSSs locations, and (iv) inclusion of forbidden areas.

In Section II the main optimization problem is formulated including objective function and constraints definition. Later on in the Section III the modelling aspects are explained in detail, followed by the whole framework description in Section IV. Computational experiments are performed in Section $[\mathrm{V}$ and the work is finalized with the conclusions in Section VI

\section{Optimization MODEL}

In this manuscript is developed a model that supports any reasonable number of WTs, $n_{w}$, and any number of OSSs, $n_{o}$. Define the set $\mathcal{N}_{o}=\left\{\mathcal{N}_{o 1}, \cdots, \mathcal{N}_{o n_{o}}\right\}$, where an element $\mathcal{N}_{o i}$ is, in turn, an index set representing candidate locations for the $i$ th OSS with $i \leq n_{o}$. In this way, $\beta_{t}=\sum_{1 \leq i \leq n_{o}}\left|\mathcal{N}_{o_{i}}\right|$ defines the total number of potential locations to place OSSs (denoted $\mathcal{N}_{\beta_{t}}=\left\{1, \cdots, \beta_{t}\right\}$ ), given a required number $n_{o}$, and individual candidate set, $\mathcal{N}_{o i}$, for each $i \in \mathcal{N}_{n_{o}}=\left\{1, \cdots, n_{o}\right\}$. Likewise, let $\mathcal{N}_{w}=\left\{\beta_{t}+1, \cdots, \beta_{t}+n_{w}\right\}$ denote the index set representing the WTs. Hence, the whole set of points including both OSSs candidate locations and WTs locations is given as, $\mathcal{N}=\{0\} \cup \mathcal{N}_{\beta_{t}} \cup \mathcal{N}_{w}$, where the node 0 represents the OCP.

The aim is to obtain the optimum design of the cable layout for a full OWF, modelled as $n_{o}$ spanning-trees, connecting the WTs between each other, and towards the desired number 
of OSSs, along with the transmission cables from OSSs to the OCP. Furthermore, as OWFs cover a relatively large area, it is common that they include forbidden zones - defined as spaces where no WTs or cables can be placed - which must be considered as well. The geographical location of the WTs is fixed, calculated in the micrositing optimization process in order to maximize the energy produced in the OWF [18]. The distance between two points $i$ and $j$, is defined as $d_{i j}$.

The complete directed graph $G(\mathcal{N}, \mathcal{A}, \mathcal{D})$ comprises the input sets, where $\mathcal{N}$ represents the vertex set, $\mathcal{A}$ the set of available arcs arranged as a pair-set, and $\mathcal{D}$ the set of associated weights for each element $a \in \mathcal{A}$.

The different types of cables being considered are stored in $\mathcal{T}_{c}$, and $\mathcal{T}_{e}$, for the collection and export systems, respectively. The set $\mathcal{T}_{c}$ relates to the attribute sets $\mathcal{U}_{c}$, and $\mathcal{C}_{c}$, representing the capacity (in terms of maximum number of supported WTs), and total capital expenditures per unit of length, respectively. In general, the greater the cable capacity, the greater the capital cost.

The first set of variables in the model are binary: $z_{i, l} x_{i j}$, and $y_{i j}^{k}$. Choosing the candidate location $l$ for the OSS $i$ is done through the variable $z_{i, l}$, which is equal to one if selected. and zero otherwise. If the arc connecting $i$, and $j$ is active $(i \in \mathcal{N} \backslash\{0\} \wedge j \in \mathcal{N} \backslash\{0\})$, then $x_{i j}=1$. Finally, in case $x_{i j}=1$, the number of WTs connected is defined by $y_{i j}^{k}$, where $k$ models the number of WTs rooted at $i$, including the one at $j$. A set of positive integer variables is required as well; $\sigma_{i}$, and $\sigma_{i, l}$ represents the number of WTs connected to the OSS $i$, and at the corresponding candidate location $l$, respectively.

The number of $x_{i j}$, and $y_{i j}^{k}$ variables scale quadratically with the number of WTs. Therefore, reduction strategies are important to limit the size of the model in terms of variables and constraints. A procedure proposed in [19] and [17] is used. The largest individual cable capacity is found as $U_{c}=\max \mathcal{U}_{c}$; consequently, the maximum attributable value of $k$ for $i \in \mathcal{N}_{\beta_{t}}$ is equal to $f(i)=U_{c}$, in contrast, for elements $i \in \mathcal{N}_{w}^{t}$ is $f(i)=U_{c}-1$. This is because, intuitively, a cable only can be used at maximum capacity if connected to a OSS. Finally, all redundant arcs are suppressed, along with those interconnecting OSSs, as only the common industry practice of point-to-point connections from offshore to onshore points is considered. Overall, the original graph $G(\mathcal{N}, \mathcal{A}, \mathcal{D})$ is reduced to $G_{r}\left(\mathcal{N}, \mathcal{A}_{r}, \mathcal{D}_{r}\right)$.

\section{A. Cost coefficients}

Designing the electrical cables system in OWFs is manifold: not only arcs must be selected while also choosing the cable type to do so, but total electrical power losses must be considered as they may impact the design. Total electrical power losses are function of the selected arc, cable type, and generated power.

Hence, the straight-forward way to include simultaneously all these aspects is to incorporate in the variable $y_{i j}^{k}$ the cable type, as for instance transforming the variable to $y_{i j}^{k, t}$. Variable $y_{i j}^{k, t}$ would model whether the arc $a_{r}=(i, j)$ is selected or not, connecting $k$ WTs through cable type $t$. Secondly, losses would have to be mathematically explicitly expressed in the objective function. This raises two issues: (i) the number of variables increase linearly with the number of cable types $\left|\mathcal{T}_{c}\right|$, and (ii) ohmic losses are non-linear, therefore simplifications must be assumed for expressing it in a linear model.

A body of actions to circumvent these limitations, while being able to solve the defined problem, are implemented. The approach basically decouples the arc selection and losses minimization from the cable assignment decision-making problem. These techniques are explained in the following sections for both the collection system (cable layout), and transmission system (point-to-point connection).

1) Collection system cables: The reduced graph $G_{r}\left(\mathcal{N}, \mathcal{A}_{r}, \mathcal{D}_{r}\right)$ contains all required information for the cable layout. For $y_{i j}^{k}$, the length of arc $(i, j)$ is known, along with the number of WTs connected; this makes possible to evaluate the whole set of available cables in polynomial running time for each of the $\operatorname{arcs}$ (such as $i \neq 0 \wedge j \neq 0$ ), to select that which minimizes the objective function. All cost coefficients are non-negative.

Let the term $\left(c_{c_{t}} \cdot d_{i j}\right)$ represent the capital expenditures plus installation costs (per metric unit) of cable $t$, to join points $i$ and $j$ (cost per metric unit). The parameters $c_{c_{t}} \in \mathcal{C}_{c}$ are obtained from the exponential regression function given in [20] (excluding installation costs).

To incorporate the cost of total electrical power losses, a discounted cash flow metric is considered. The required parameters are $|\mathcal{M}|, l_{c_{i j, t}}^{\mu, k}, c_{p}$, and $r$, meaning the project lifetime (years), total power losses at year $\mu$ for cable $t$, when $k$ WTs are connected (in MWh, see Section III-C), cost of energy ( $€ / \mathrm{MWh}$ ), and discount rate (p.u.), respectively.

Bearing this in mind, for each $(i, j)$ such as $i \neq 0 \wedge j \neq 0$, the following optimization model is formulated and solved independently by enumeration [17].

$$
\begin{array}{cc}
c_{i j}^{k}=\min & \sum_{t \in \mathcal{T}_{c}} x_{c_{i j, t}} \cdot\left(c_{c_{t}} \cdot d_{i j}+\sum_{\mu=1}^{|\mathcal{M}|} \frac{l_{c_{i j, t}, k}^{\mu, c_{p}}}{(1+r)^{\mu}}\right) \\
\text { s.t. } & \sum_{t \in \mathcal{T}_{c}} x_{c_{i j, t}}=1 \\
& x_{c_{i j, t}} \cdot\left(S_{c_{i j, t}}^{k}-S_{r c_{t}}\right) \leq 0 \quad \forall t \in \mathcal{T}_{c} \\
x_{c_{i j, t}} \in\{0,1\} \quad \forall t \in \mathcal{T}_{c}
\end{array}
$$

Note that this set of problems are always feasible as $k$ is limited by $U_{c}$. Equation (2) ensures that exactly one cable type is selected. Equation (3) guarantees that the capacity of cable $t$ is not violated; where $S_{c_{i j, t}}^{k}$ is the power through arc $(i, j)$, when $k$ turbines are connected in $j$ using cable $t$, and $S_{r c_{t}}$ is the rated power of $t$ (see Section III-A and Section III-B. Lastly, Equation (4) defines the nature of the problem's variables.

After solving the model from (1) to (4) (maximum $U_{c} \cdot|\mathcal{N}|^{2}$ times), the cost coefficients $c_{i j}^{k}$ are calculated, and the corresponding cable type $t$ is unequivocally determined as per $x_{c_{i j, t}}$.

2) Transmission system cables: Arcs $(i, j)$ such as $i \in\{0\}$ $\wedge j \in \mathcal{N}_{\beta_{t}}$ are also definable beforehand. The cost of the transmission cables is function of the total length and installed 
power; the former is known, but the latter is also output of the main optimization model. Likewise, in contrast to the collection system cable layout, there are no binary variables explicitly representing whether certain number of WTs are connected to a OSS or not. In this case, the variables $\sigma_{i, l}$ are used to estimate the cost for a specific candidate location $l \in \mathcal{N}_{\beta_{t}}$, associated to the OSS $i \in \mathcal{N}_{n_{o}}$.

Let the variable $x_{0 j}^{t, n}$ represent if arc $(0, j)$ is active using $n$ cables $t$ in parallel or not (a limit of $n=3$ is a reasonable practical constraint to ensure feasibility). The methodology uses the following optimization model, where the target is to obtain a linear cost function in terms of the WTs connected to a OSS:

$$
\begin{array}{cc}
\min \quad \sum_{n=1}^{3} \sum_{t \in \mathcal{T}_{e}} x_{e_{i j, t, n}} \cdot\left(c_{e_{t, n}} \cdot d_{0 j}+\sum_{\mu=1}^{|\mathcal{M}|} \frac{l_{e_{0 j, t, n}}^{\mu, k} \cdot c_{e}}{(1+r)^{\mu}}\right) \\
\text { s.t. } \\
\sum_{n=1}^{3} \sum_{t \in \mathcal{T}_{e}} x_{e_{i j, t, n}}=1 \\
\sum_{n=1}^{3} x_{e_{i j, t, n}} \cdot\left(S_{e_{0 j, t, n}}^{k}-S_{r e_{t}}\right) \leq 0 \quad \forall t \in \mathcal{T}_{e} \\
x_{e_{i j, t, n}} \in\{0,1\} \quad \forall t \in \mathcal{T}_{e} \wedge n \in\{1,2,3\}
\end{array}
$$

The mathematical program from (5) to (8) resembles that described from (1) to (4). In fact, each equation is mirrored by the order of appearance. Nevertheless, in the case of the transmission cables, most of the parameters and variables are re-indexed accounting for $n$, which is the number of cables per connection. Contrary to collection system cables, in transmission level parallel cables are installed in practice. Figure 11 presents the basic algorithm for calculating the transmission cables linear cost function. The idea behind is to sequentially increase the number $k$ of turbines connected to a specific OSS location, solving (5) to (8), and gathering all results to finally obtain the best linear function fitting the data by least squares method.

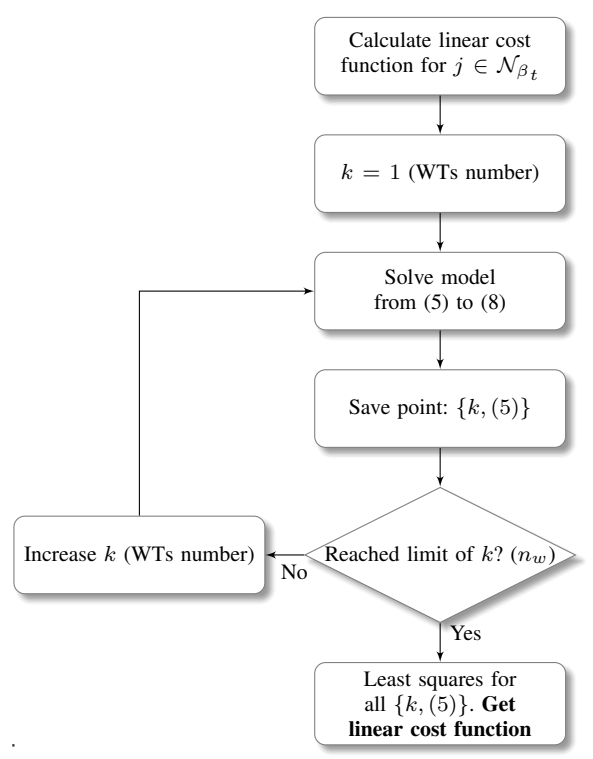

Fig. 1: Flowchart for calculating the transmission cables linear cost function.

The procedure is performed for each OSS candidate location.
Thus, $\beta_{t}$ linear functions are obtained, each characterized by a independent $c_{l}^{o}$, and non-negative linear $c_{l}^{\iota}$ term, where $l \in \mathcal{N}_{\beta_{t}}$.

By adaptations on the costs coefficients in (1) and (5), the following objective functions are supported for the global model: length (L), length plus total power losses (LP), initial investment (I), and initial investment plus total power losses (IL)

\section{B. Objective function}

After solving the collection and transmission systems sub-problems, the linear objective function of the main mathematical model is formulated as:

$$
\begin{aligned}
& \min \sum_{i \in \mathcal{N} \backslash\{0\}} \sum_{j \in \mathcal{N}_{w}} \sum_{k=1}^{f(i)} c_{i j}^{k} \cdot y_{i j}^{k} \\
& +\sum_{i \in \mathcal{N}_{n_{o}}} \sum_{l \in \mathcal{N}_{o i}}\left(c_{l}^{o} \cdot z_{i, l}+c_{l}^{\iota} \cdot \sigma_{i, l}\right)
\end{aligned}
$$

\section{Constraints}

For a given set of candidate locations $\mathcal{N}_{o i}$ for the OSS $i$, exactly one of them must be chosen. This is modelled through

$$
\sum_{l \in \mathcal{N}_{o i}} z_{i, l}=1 \quad \forall i \in \mathcal{N}_{n_{o}}
$$

Note that in constraint (10), if an inequality (less than or equal to) replaces the equality, not only multiple locations would be supported, but also multiple number of OSSs.

In order to cluster the WTs $\left(n_{w}\right)$ into $n_{o}$ OSSs, the following constraint is required:

$$
\sum_{i \in \mathcal{N}_{n_{o}}} \sigma_{i}=n_{w}
$$

In (11), $\sigma_{i}$ represents the number of WTs associated to the OSS $i$, its definition is:

$$
\sum_{l \in \mathcal{N}_{o i}} \sigma_{i, l}=\sigma_{i} \quad \forall i \in \mathcal{N}_{n_{o}}
$$

Correspondingly, $\sigma_{i, l}$ counts the number of WTs connected to the OSS $i$ at location $l$ and is computed through

$$
\sum_{j \in \mathcal{N}_{w}} \sum_{k=1}^{f(j)} k \cdot y_{l j}^{k}=\sigma_{i, l} \quad \forall i \in \mathcal{N}_{n_{o}} \wedge l \in \mathcal{N}_{o i}
$$

The variables $\sigma_{i, l}$ are for linearization, while $\sigma_{i}$ is for the model readability. The following equation, for both selecting an OSS location $z_{i, l}$ and limiting the number of feeders out from them to $\phi$, is added:

$$
\sum_{j \in \mathcal{N}_{w}} \sum_{k=1}^{f(j)} y_{l j}^{k} \leq \phi \cdot z_{i, l} \quad \forall i \in \mathcal{N}_{n_{o}} \wedge l \in \mathcal{N}_{o i}
$$

The tree topology, i.e. only one cable type used per arc, and the definition of the head-tail convention, are simultaneously ensured by:

$$
\sum_{i \in \mathcal{N} \backslash\{0\}} \sum_{k=1}^{f(i)} y_{i j}^{k}=1 \quad \forall j \in \mathcal{N}_{w}
$$


The flow conservation, which also avoids the formation of cycles (loops), is considered by means of one linear equality per wind turbine

$$
\sum_{i \in \mathcal{N} \backslash\{0\}} \sum_{k=1}^{f(i)} k \cdot y_{i j}^{k}-\sum_{i \in \mathcal{N}_{w}} \sum_{k=1}^{f(i)} k \cdot y_{j i}^{k}=1 \quad \forall j \in \mathcal{N}_{w}
$$

The set $\mathcal{I}$ stores pairs of arcs $\{(i, j),(u, v)\}$, which are crossing each other. Excluding crossing arcs in the solution is ensured by the linear inequalities

$$
\begin{gathered}
x_{i j}+x_{j i}+x_{u v}+x_{v u} \leq 1 \\
\forall\{(i, j),(u, v)\} \in \mathcal{I}:\{i, j, u, v\} \neq 0 \\
\sum_{k=1}^{f(i)} y_{i j}^{k}-x_{i j} \leq 0 \quad \forall(i, j) \in \mathcal{A}_{r}:\{i, j\} \neq 0
\end{gathered}
$$

Constraint (17) also includes the inverse arcs of those elements. This constraint is a practical restriction in order to avoid hot-spots and potential single-points of failure caused by overlapped cables [21].

The following constraints represent a set of valid inequalities to tighten up the mathematical model [22]:

$$
\begin{gathered}
-\sum_{i \in \mathcal{N} \backslash\{0\}} \sum_{k=v+1}^{f(i)}\left\lfloor\frac{k-1}{v}\right\rfloor \cdot y_{i j}^{k}+\sum_{i \in \mathcal{N}_{w}} \sum_{k=v}^{f(i)} y_{j i}^{k} \leq 0 \\
\forall v \in\left\{2, \cdots, U_{c}-1\right\} \wedge j \in \mathcal{N}_{w}
\end{gathered}
$$

They can be interpreted as: given an active arc $y_{i j}^{k}$, the maximum number of active arcs rooted in $j$ and connecting $v$ WTs is expressed by $\left\lfloor\frac{k-1}{v}\right\rfloor$, hence the constraint restricts the maximum number of feasible arcs, reducing the search space without excluding valid solutions to the problem.

$$
\begin{gathered}
x_{i j} \in\{0,1\} \quad y_{i j}^{k} \in\{0,1\} \\
\forall(i, j) \in \mathcal{A}_{r}:\{i, j\} \neq 0 \wedge k \in\{1, \cdots, f(i)\} \\
z_{i, l} \in\{0,1\} \quad 0 \leq \sigma_{i} \leq \eta \cdot\left\lceil\frac{n_{w}}{n_{o}}\right\rceil \quad \sigma_{i} \wedge \sigma_{i, l} \in \mathbb{Z}_{+} \\
\forall i \in \mathcal{N}_{n_{o}} \wedge l \in \mathcal{N}_{o i}
\end{gathered}
$$

Constraints (20) and (21) define the nature of the formulation by the variables definition, a MILP. Note that variables $\sigma_{i}$ are limited in their upper bounds to avoid unbalanced OSSs, in terms of connected WTs (in case $\eta=1$, if unbalancing is permitted then $1<\eta \leq n_{o}$ ), which implicitly also bounds $\sigma_{i, l}$.

To summarize, the complete formulation of the main MILP model consists of the objective function (9) and constraints defined in (10) - 21.

\section{MODELLING ASPECTS}

The modelling choices presented in the following - with the exception of Section III-D - are based on the ones presented in [17] (particularly Section III-A, Section III-B, and Section III-C).

\section{A. Cable capacity}

The current capacity $I_{c_{t}}$ of a cable $t$ is calculated using the model given in [23]. This method comes from a Single-Core Equivalent Thermal Model (SCETM), as generalized in [24] for single-core and three-core cables. It is conservative when applied for OWFs, hence lifetime of the component is within acceptable intervals [25].

$$
\begin{aligned}
& u_{c_{t}}=\left\lfloor\frac{S_{r c_{t}}=\sqrt{3} \cdot V_{n_{c}} \cdot I_{c_{t}}}{P_{n}}\right\rfloor \quad \forall t \in \mathcal{T}_{c} \\
& \mathcal{U}_{c}=\left\{1, \cdots, u_{c_{\left|\tau_{c}\right|}}\right\}
\end{aligned}
$$

The set of cable capacities in terms of number of supportable WTs is defined in (22) and (23), where $P_{n}$ represents the nominal power of an individual WT, and $V_{n_{c}}$ the nominal lineto-line voltage level in the collection system. Regarding the transmission system, the rated power of cables $\left(S_{r e_{t}} \forall t \in \mathcal{T}_{e}\right)$ is calculated with the voltage level $V_{n_{e}}$, such as $S_{r e_{t}}=\sqrt{3}$. $V_{n_{e}} \cdot I_{e_{t}}$.

\section{B. Arcs nominal power}

A distributed model is implemented with the next expressions [26]

$$
\begin{gathered}
I_{c_{i j, t}}^{\vec{k}}=\frac{k \cdot P_{n}}{\sqrt{3} \cdot V_{n_{c}}} \cdot \cosh \left(\overrightarrow{\gamma_{t}} \cdot d_{i j}\right)-\frac{V_{n_{c}}}{\overrightarrow{Z_{c_{t}}}} \cdot \sinh \left(\overrightarrow{\gamma_{t}} \cdot d_{i j}\right) \\
S_{c_{i j, t}}^{k}=\sqrt{3} \cdot V_{n_{c}} \cdot\left|I_{c_{i j, t}}^{\vec{k}}\right|
\end{gathered}
$$

The characteristic impedance is calculated as $\overrightarrow{Z_{c_{t}}}=\sqrt{\overrightarrow{z_{t}} / \overrightarrow{y_{t}}}$, and the propagation constant, $\overrightarrow{\gamma_{t}}=\sqrt{\overrightarrow{z_{t}} \cdot \overrightarrow{y_{t}}}$. The series impedance is represented by $\overrightarrow{z_{t}}$, and the admittance by $\overrightarrow{y_{t}}$. The maximum power flowing through the arc $(i, j)$, given $k$ turbines connected downstream $\left(y_{i j}^{k}\right)$, is calculated as per 25, , accounting for the worst-case scenario, as the current in the arc is strictly increasing with length, and the value $I_{c_{i j, t}}^{\vec{k}}$ is calculated at the extreme of it when using cable $t$.

For the transmission cables, (24) and (25) must be calculated per cable $\left(\frac{k \cdot P_{n}}{n_{\rightarrow}}\right)$, and considering $V_{n_{e}}$, to obtain $S_{e_{i j, t, n}}^{k}=$ $\sqrt{3} \cdot V_{n_{e}} \cdot\left|I_{e_{i j, t, n}}^{k \overrightarrow{ }}\right|$.

\section{Power flow and total power losses}

A transportation model, accurate enough for radial systems, is implemented through Constraint 16. Time variation can be represented as:

$$
\begin{gathered}
\sum_{i \in \mathcal{N}} \sum_{k=1}^{f(i)} k \cdot y_{i j}^{k} \cdot p^{\omega}-\sum_{i \in \mathcal{N}_{w}} \sum_{k=1}^{f(i)} k \cdot y_{j i}^{k} \cdot p^{\omega}=p^{\omega} \\
\forall j \in \mathcal{N}_{w} \wedge \omega \in \Omega^{\mu} \wedge \mu \in \mathcal{M}
\end{gathered}
$$

Constraint (16) is generalized considering the temporal dimension $[26$, where $\mathcal{M}=\{1, \cdots,|\mathcal{M}|\}$, is the set of operational years with upper limit in the project lifetime, $\Omega^{\mu}$ is the set of hours-slot for a year $\mu$, and $\omega$ a specific hour-slot in $\Omega^{\mu}$. By means of the simulation of offshore power time series [27], let $p^{\omega}$ be the power in MW produced by one WT in that hour-slot. 
In this way, let define the auxiliary variable $f_{i j}^{\omega, k}=k \cdot y_{i j}^{k} \cdot p^{\omega}$ as the power flow (MW) in arc $(i, j)$ when $k$ WTs are connected downstream (including the one in $j$ ), in time instant $\omega$.

$$
\begin{aligned}
& I_{c_{i j, t}}^{\overrightarrow{\omega, k}}=\frac{f_{i j}^{\omega, k}}{\sqrt{3} \cdot V_{n_{c}}} \cdot \cosh \left(\overrightarrow{\gamma_{t}} \cdot d_{i j}\right)-\frac{V_{n_{c}}}{\overrightarrow{Z_{c_{t}}}} \cdot \sinh \left(\overrightarrow{\gamma_{t}} \cdot d_{i j}\right) \\
& l_{c_{i j, t}}^{\mu, k} \approx 3 \cdot\left(1+\lambda_{1}+\lambda_{2}\right) \cdot \sum_{\omega \in \Omega^{\mu}} \omega \cdot R_{t} \cdot d_{i j} \cdot\left|I_{c_{i j, t}}^{\overrightarrow{\omega, k}}\right|^{2} \\
& +3 \cdot\left|\Omega^{\mu}\right| \cdot \omega \cdot W_{d_{t}}
\end{aligned}
$$

Including the capacitive currents, 27) expresses the current at the end of the arc (with respect to $i$ ), with magnitude $\left|I_{i j}^{\vec{\omega}, k}\right|$. The annual total power losses $l_{c_{i j, t}}^{\mu, k}$ is calculated with 28). The factor $\left(1+\lambda_{1}+\lambda_{2}\right)$ accounts for the screen and armouring losses (with values from [23]), $W_{d_{t}}$ is the dielectric loss per unit length for the insulation surrounding the conductor in $\mathrm{W} / \mathrm{m}$ (formula given in [23]), while the constant 3 is for the three-phase system. This value must be scaled in MWh. Losses in transmission cables $\left(l_{e_{i j, t, n}}^{\mu, k}\right)$ must consider the number of components in paralell per phase.

\section{Forbidden areas}

A restricted set of regions (inside a OWF) for excavation and cable trenching is supported in this model. Those regions can be modelled as convex polygons [28], non-convex polygons [29], or closed curves [30]. A convex hull based bypassing algorithm which may incorrectly delimit areas as forbidden, if they are non-convex polygons, is proposed in [28]. Any polygonal shape can be defined using Steiner nodes explicitely in the model as in [29], where the aim is not only to model the area, but also to refine arcs with shortest path. However, bending moments in the cables may be compromised as a result of unrealistic routing. Finally, more accurate computational algorithms to represent more precisely defined shapes is implemented in [30], by means of Delaunay Triangulation Based Navigational Mesh Path-finding. Despite this, unrealistic routing can result as well.

A pre-processing strategy schematized in Fig. 2 is proposed in this manuscript. Any polygonal shape is modelled, while simultaneously decreasing the number of variables. By means of this approach, the explicit creation of variables and constraints in the main model to cope with forbidden areas is avoided, while allowing only straight arcs between WTs. On the other hand, the strategy is flexible as any number of shapes are supported.

Let the set of forbidden areas be $\mathcal{L}$. An area $\ell \in \mathcal{L}$, in turn, is defined as a set of arcs $\mathcal{A}_{\ell}$, where $b \in \mathcal{A}_{\ell}: b=$ $(i, j)$. The set of arcs $\mathcal{A}_{\ell}$ defines a sequence of vertices enveloping the points representing a forbidden area $l$ (the sequence $\{i, j, v, i\}$ represents, for example, a triangle with $\left.\mathcal{A}_{\ell}=\{(i, j),(j, v),(v, i)\}\right)$. The procedure for incorporating these zones into the model is depicted in Fig. 2, where graph $G_{r}^{\prime}$ is obtained from graph $G_{r}$, by excluding from the new $\operatorname{arcs}$ set $\mathcal{A}_{\nabla}^{\prime}$ all the arcs crossing with at least one arc $b \in \mathcal{A}_{\ell}$.

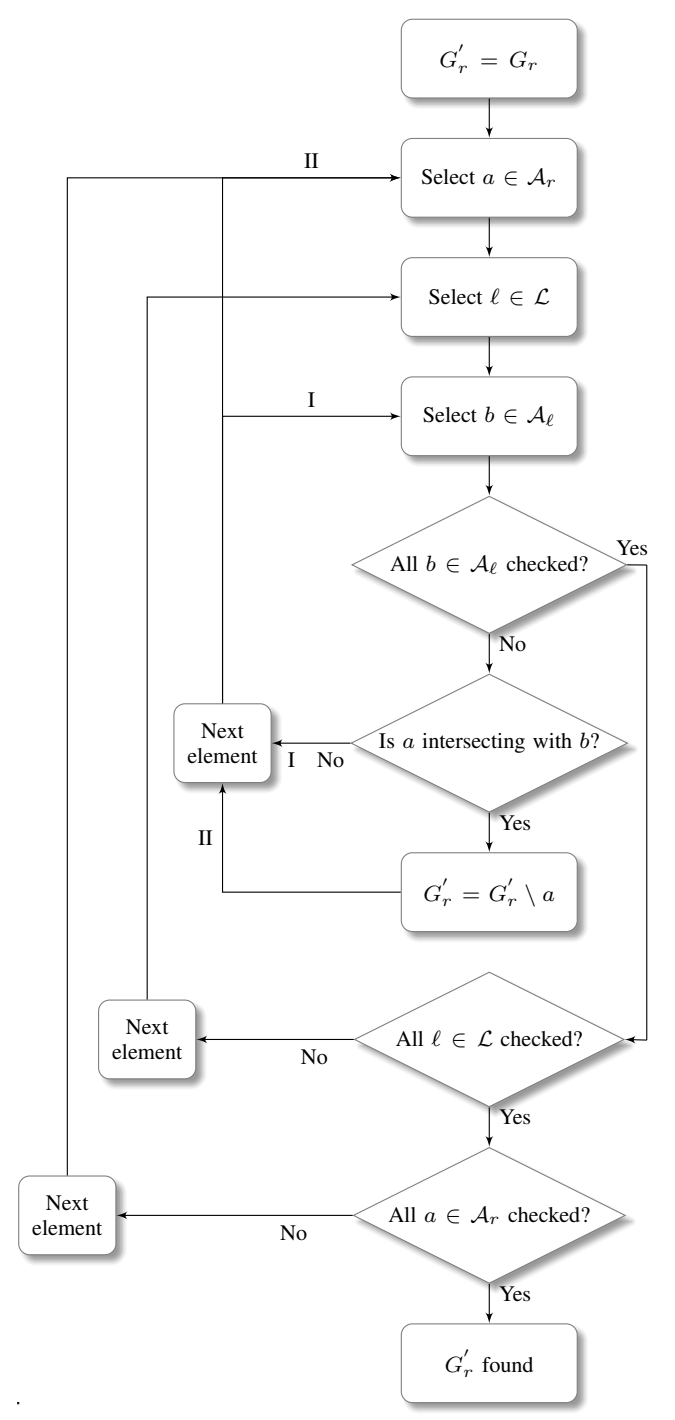

Fig. 2: Flowchart for incorporating forbidden areas in the model.

\section{OPTIMIZATION FRAMEWORK}

For large OWFs, i.e. in the range of 100s of WTs, the cable layout problem is generally unsolvable for gaps lower than $1 \%$ [31]. This is also the authors' computational experience. Therefore the graph $G_{r}^{\prime}$ needs further reduction to make the model tractable.

The framework proposed in [17] is generalized in this work. Let the function $f\left(i, G_{r}^{\prime}, v\right)$ obtain the set $\Upsilon_{i}$ defined as maximum the $v$-closest WTs to $i$; the term maximum accounts for vertices which have less than $v$ arcs available due to prior elimination for crossings with forbidden areas. In other words, it is considered intuitively that a WT will be connected to one of the WTs in its vicinity. Therefore, by systematically applying $f\left(i, G_{r}^{\prime}, v\right)$ to each $i \in \mathcal{N}_{w}$, the reduced graph $G_{r}^{\prime \prime}$ is found. The set $\mathcal{A}_{\nabla}^{\prime \prime}$ contains the candidate arcs to the solution of the problem. The outcoming arcs from OSSs are also limited to the nearest 50 WTs. An imposed gap of $\epsilon$ is an input of the method.

The flowchart is displayed in Fig. 3, as a first step a feasibility problem is solved, characterized by (i) an objective function 
equal to zero, (ii) a low $v$ is set to speed up the process, and (iii) fixed locations of OSSs by choosing arbitrarily one per each $i$ available in $\mathcal{N}_{o i}$. Following the solution of the

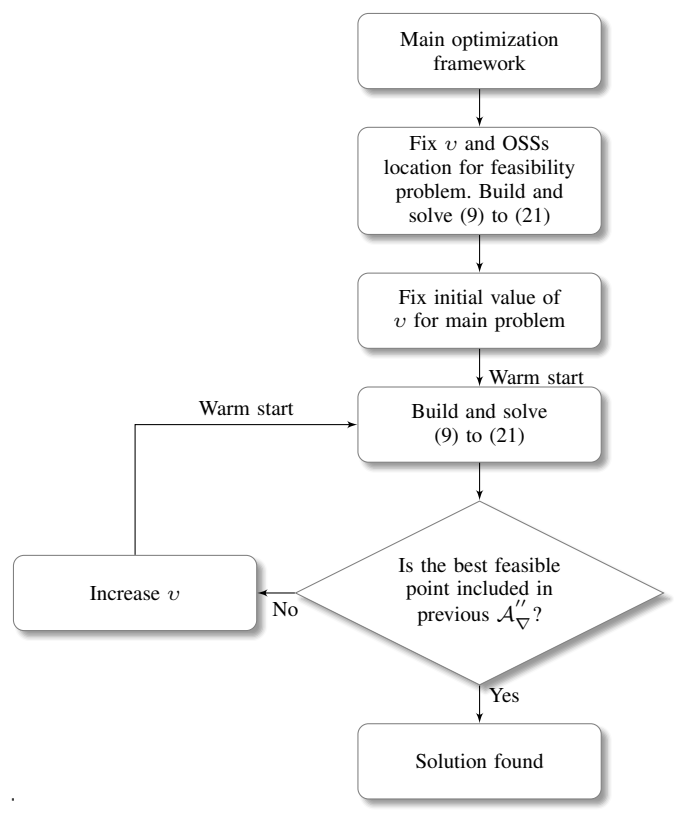

Fig. 3: Flowchart of the main optimization framework.

feasibility problem, the obtained solution is used to warm start the main model given $G_{r}^{\prime \prime}$ for a specific value of $v$. The next step is to compare if the best feasible point found so far, is included in the domain of the previously defined problem $\left(\mathcal{A}_{\nabla}^{\prime \prime}\right)$. If this is the case, the process is stopped, since most likely the global minimum has been calculated. Otherwise, the domain is further increased until that condition is satisfied. In the authors' earlier work [17], it has been found out that for the feasibility problem $v=5$, and for the main problem an initial value of $v=15$, and increasing steps equal to 5 , represent good parameters to find a proper compromise between computing time, and solution quality.

\section{Case Study}

The following case studies have been carried out on an Intel Core i7-6600U CPU running at $2.50 \mathrm{GHz}$ and with 16 GB of RAM. The chosen MILP solver is the branch-andcut solver implemented in IBM ILOG CPLEX Optimization Studio V12.7.1 [32].

A synthetic OWF (SY) with three forbidden areas is used to test the validity of the model. The real project London Array (LA) which is the second largest (measured by installed power) project under operation [33], is used to illustrate the ability of the model to solve very large instances. Both cases have more than 100 WTs and several OSSs, representing very challenges instances to solve.

The main high-level parameters for both case studies are given in Table II. The objective function is defined by a combined total economic cost including the initial investment, and the total electrical power losses of the cables systems (see (1) and (5)). Three forbidden areas are considered in SY, with four candidate locations per OSS, and distance to OCP of roughly $100 \mathrm{~km}$. In LA three possible locations are supported for each OSS, with length to OCP close to $20 \mathrm{~km}$. In all cases, a maximum number of 10 feeders is allowed per OSS (see (14), in addition to a balanced allocation of WTs to OSSs. Balancing of OSSs is accounted by means of $\eta=1$, see (21). The technical and economic parameters for both medium and

TABLE I

MAIN HIGH-LEVEL PARAMETERS FOR THE PROBLEM INSTANCES

\begin{tabular}{|c|c|c|c|c|c|c|}
\hline OWF & Objective & $r[\%]$ & $c_{p}[€ / \mathrm{MWh}]$ & $|\mathcal{M}|$ & $|\mathcal{L}|$ & $\eta$ \\
\hline $\begin{array}{l}\text { SY } \\
\text { LA }\end{array}$ & IL & 5 & 40 & 30 & $\begin{array}{l}3 \\
0 \\
\end{array}$ & 1 \\
\hline OWF & $P_{n}[\mathrm{MW}]$ & & $\mathcal{N}_{o}$ & $n_{w}$ & $n_{o}$ & $\phi$ \\
\hline $\begin{array}{l}\text { SY } \\
\text { LA }\end{array}$ & 3.6 & $\begin{array}{l}\{\{1,2 \\
\quad\{\{1\end{array}$ & $\begin{array}{l}4\},\{5,6,7,8\}\} \\
3\},\{4,5,6\}\}\end{array}$ & $\begin{array}{l}120 \\
175\end{array}$ & 2 & 10 \\
\hline
\end{tabular}

high voltage cables are presented in Table $\Pi$. Further technical cable information is available in [34]. For the collection system, the set of cable dimensions is $240 \mathrm{~mm}^{2}, 500 \mathrm{~mm}^{2}$, and $1,000 \mathrm{~mm}^{2}$, while for transmission, ten different cables types are considered, ranging from $300 \mathrm{~mm}^{2}$ to $2,000 \mathrm{~mm}^{2}$. All the cross-sections are commercially standards by manufacturers. Cable capacities and costs are displayed for reproducibility as well in Table III. The linear cost function obtained using Fig.

TABLE II

CABLES MAIN PARAMETERS

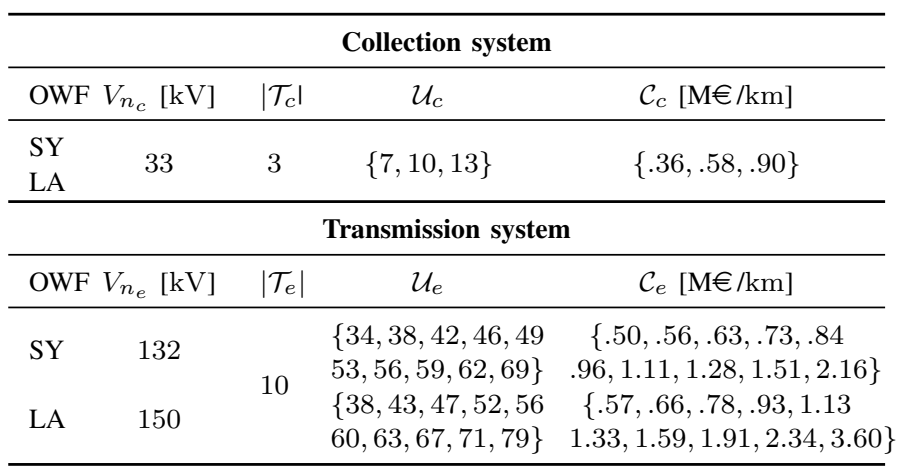

11 for the first candidate location of OSS 1 in SY OWF is presented in Fig. 4 with a distance to shore of $101.31 \mathrm{~km}$. In this graph two set of curves are displayed. The first one (blue circles and line) includes the optimization objective function output when (5) - (8) are solved neglecting total electrical power losses (economic losses' discounted cash flow) in (5). The blue circles are the optimum cost of transmission cables from (5), while the blue line is the best linear curve fitting that data. In contrast, the second set of curves is determined by including the total electrical power. The red circles are from the optimization objective function, and the red line is the best linear data fitting.

Three main outcomes can be extracted from Fig. 4 . (i) total electrical power losses considerably boost the total costs. For 55 WTs an increase of $50 \%$ in costs is estimated. (ii) including electrical losses improves the linear function fit, as the $R^{2}$ value of the linear data fitting calculated with losses is higher 


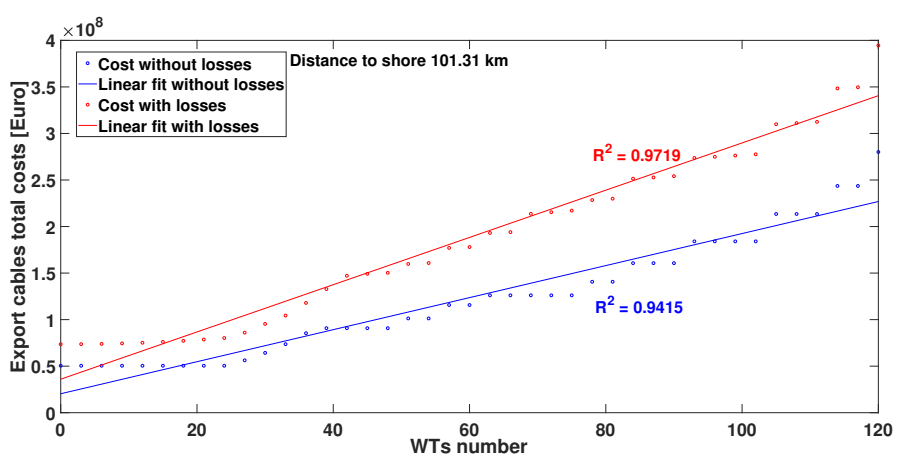

Fig. 4: Linear cost function with and without total electrical power losses.

than that without $(0.9719>0.9415)$. This is because without taking electric losses into account, a larger number of WTs can be connected to the same cable size. The export route length for different OSSs candidate locations affects mainly the slope of the corresponding linear function, thus, the longer the length the higher the slope. Finally, (iii), it can be seen for both sets that for low WTs numbers, i.e. between 1 and 40, the transmission cables minimum cost is poorly represented by the linear functions. However, it improves visibly at around 40 WTs and higher, which indicates that it can be used for the OWFs target size considered in this manuscript.

Overall, it is possible to infer that by including economic costs of electrical losses, not only a more holistic approach is supported (losses impact the layout as shown in [4], [35]), but also a statistical improvement is achieved, which enhances the accuracy of the model. The graphical result of applying the integrated global optimization model for electrical cables in SY OWF is presented in Fig. 5 Candidate locations are indicated with red numbers. All hard constraints are satisfied, along with the non crossing of forbidden areas. Only cables with 240 and $500 \mathrm{~mm}^{2}$ are used in the collection system. For the OSSs, the locations closest to the OCP are chosen, hence minimizing the transmission cable length. Each OSS is being connected with two $500 \mathrm{~mm}^{2}$ cables.

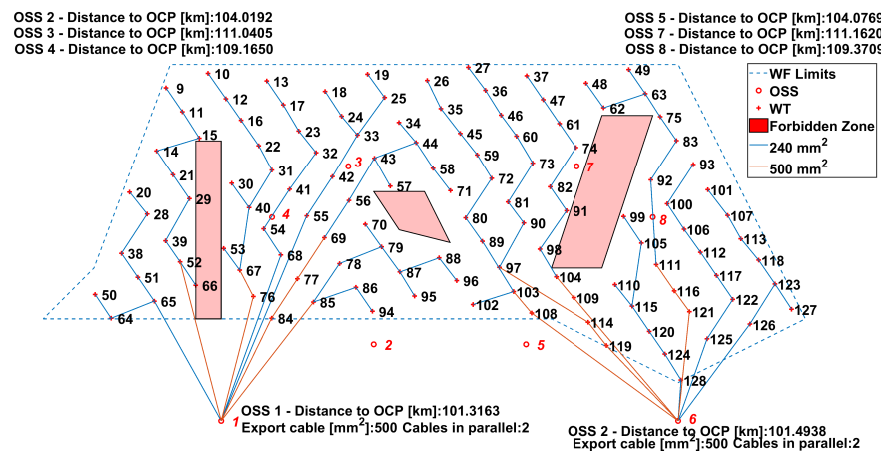

Fig. 5: SY OWF designed cable layout

Numerical results are available in Table III For SY OWF $(\epsilon=0.5 \%)$ an optimality gap of $0.49 \%$ is achieved in 3 hours, and 31 minutes. An initial feasible solution is found in 81 seconds, with optimality gap equal to $13.42 \%$. In order to assess if the solver is able to take advantage of the problem's structure, an instance of SY, called as SYs, where only one candidate location is fixed per OSS (number 1 and 6 in Fig. 5), is solved by the model. An equal solution is obtained in 1 hour, and 28 minutes. The total number of combinations in terms of OSSs pairs is 16 . If one assumes this an average running time, then a total of 23 hours, and 31 minutes would be required following an enumeration approach. By means of the integrated model a reduction of $85 \%$ of running time is obtained.

TABLE III

MAIN RESULTS SUMMARY

\begin{tabular}{cccc}
\hline Model & Best solution [M€] & Gap [\%] & Total computation time [h] \\
\hline SY & 487.47 & 0.49 & 3.53 \\
SYs & -487.76 & 0.50 & 1.48 \\
$-\overline{\text { LA }}---$ & $-\overline{14} \overline{4}---$ & $0 . \overline{7} 5$ & --- \\
\hline
\end{tabular}

Finally, the very large instance of LA OWF is tackled. The two previously described instances have been solved using the default settings offered by CPLEX. However, out-of-memory problems were faced when evaluating the LA instance. To cope with this limitation, strong variable selection is used. This means that CPLEX invests considerable effort in analyzing potential branches of the nodes tree in the hope of drastically reducing the number of nodes that are explored. The strategy compromises the running time but generally allows for less memory requirements. The LA OWF is solved with a gap of $0.75 \%$ in slightly more than 23 hours. In this case, the optimal solution results in OSSs located around the center of the OWF. This is intuitively valid, as the shorter length to shore (around $20 \mathrm{~km}$ ) moves the weight towards minimizing the total length of the collector system cables.

\section{CONCLUSION}

The proposed program provides an integrated global optimization approach to design electrical cable systems of OWFs, particularly the collection and transmission systems. The contributions of this manuscript are manifold: (i) capability to harmonize and design both systems through a MILP model, (ii) fast computing time, and (ii) integration of realistic and high-fidelity physical models. The methodology has been validated against large-scale instances of OWFs projects. Several numerical results prove the validity and accuracy of the approach in terms of abiding hard constraints within reasonable computation time, considering the complexity of the problems. A synthetic OWF (SY), and the second largest project in operation today, the London Array (LA), considering three candidate locations per OSS, have been used as case studies. The SY OWF is solved in around 3.5 hours with an optimality gap of $0.49 \%$. The integrated model is roughly $85 \%$ faster than an enumeration approach. In the case of LA OWF, strong variable selection is used in the branch-and-cut method, achieving an optimality gap of $0.75 \%$ in slightly more than 23 hours. The results indicate that the physical optimal locations of the OSSs are strongly affected by the distance to shore, as the export cables total costs start becoming predominant for larger distances. 


\section{REFERENCES}

[1] GWEC, "Global Wind Report 2018," Tech. Rep., 2019. [Online]. Available: https://www.tureb.com.tr/files/bilgi_bankasi/dunya_res_ durumu/gwec_global_wind_report_2019.pdf.[AccessedMay27,2019]

[2] X. Sun, D. Huang, and G. Wu, "The current state of offshore wind energy technology development," Energy, vol. 41, no. 1, pp. 298-312, 2012.

[3] RenewableUK, "RenewableUK - Project Intelligence," 2018. [Online]. Available: https://www.renewableuk.com/page/ProjectIntelligenceHome. [AccessedMarch3,2019]

[4] M. Fischetti and D. Pisinger, "Optimizing wind farm cable routing considering power losses," European Journal of Operational Research, vol. 270, no. 3, pp. 917-930, 2018.

[5] L. Wolsey and G. L. Nemhauser, Integer and combinatorial optimization. John Wiley \& Sons, 2014.

[6] J.-A. Pérez-Rúa and N. A. Cutululis, "Electrical Cable Optimization in Offshore Wind Farms - A review," IEEE Access, vol. 7, no. 1, pp. 85 796-85 811, 2019.

[7] P. Hou, W. Hu, and Z. Chen, "Offshore substation locating in wind farms based on prim algorithm," in IEEE Power and Energy Society General Meeting, 2015, pp. 1-5.

[8] F. M. González-Longatt, P. Wall, P. Regulski, and V. Terzija, "Optimal electric network design for a large offshore wind farm based on a modified genetic algorithm approach," IEEE Systems Journal, vol. 6, no. 1, pp. 164-172, 2012.

[9] K. Deb, Optimization for engineering design: Algorithms and examples. PHI Learning Pvt. Ltd., 2012.

[10] A. Wȩdzik, T. Siewierski, and M. Szypowski, "A new method for simultaneous optimizing of wind farm's network layout and cable crosssections by MILP optimization," Applied Energy, vol. 182, pp. 525-538, 2016.

[11] S. Lumbreras and A. Ramos, "Optimal design of the electrical layout of an offshore wind farm applying decomposition strategies," IEEE Transactions on Power Systems, vol. 28, no. 2, pp. 1434-1441, 2013.

[12] P. Fagerfjäll, "Optimizing wind farm layout-more bang for the buck using mixed integer linear programming (MSc. Thesis)," Ph.D. dissertation, 2010.

[13] S. Lumbreras, A. Ramos, and S. Cerisola, "A Progressive Contingency Incorporation Approach for Stochastic Optimization Problems," IEEE Transactions on Power Systems, vol. 28, no. 2, pp. 1452-1460, 2013.

[14] M. Banzo and A. Ramos, "Stochastic Optimization Model for Electric Power System Planning of Offshore Wind Farms," IEEE Transactions on Power Systems, vol. 26, no. 3, pp. 1338-1348, 2011.

[15] P. Hou, W. Hu, Z. Chen, and C. Chen, "Overall Optimization for Offshore Wind Farm Electrical System," Wind Energy, vol. 20, no. 2016, pp. 1017-1032, 2017.

[16] O. Dahmani, S. Bourguet, M. MacHmoum, P. Guerin, P. Rhein, and L. Josse, "Optimization of the Connection Topology of an Offshore Wind Farm Network," IEEE Systems Journal, vol. 9, no. 4, pp. 1519$1528,2015$.

[17] J.-A. Pérez-Rúa, M. Stolpe, K. Das, and N. A. Cutululis, "Global Optimization of Offshore Wind Farm Collection Systems," IEEE Transactions on Power Systems (Under Review), pp. 1-12, 2019. [Online]. Available: http://arxiv.org/abs/1907.02371

[18] J. Herbert-Acero, O. Probst, P.-E. Réthoré, G. Larsen, and K. CastilloVillar, "A Review of Methodological Approaches for the Design and Optimization of Wind Farms," Energies, vol. 7, no. 11, pp. 6930-7016, 2014.

[19] A. Cerveira, A. F. de Sousa, E. J. Pires, and J. Baptista, "Optimal Cable Design of Wind Farms: The Infrastructure and Losses Cost Minimization Case," IEEE Transactions on Power Systems, vol. 31, no. 6, pp. 43194329,2016

[20] S. Lundberg, "Configuration study of large wind parks," Ph.D. dissertation, Chalmers University of Technology, 2003.

[21] J. Bauer and J. Lysgaard, "The offshore wind farm array cable layout problem: A planar open vehicle routing problem," Journal of the Operational Research Society, vol. 66, no. 3, pp. 360-368, 2015.

[22] L. Gouveia and P. Martins, "The capacitated minimum spanning tree problem: revisiting hop-indexed formulations," Computers \& operations research, vol. 32, no. 9, 2005.

[23] IEC, "IEC-60287-1: Electric cables - Calculation of the current rating," Tech. Rep., 2014. [Online]. Available: https://www.evs.ee/products/ iec-60287-1-1-2006.[AccessedApril15,2019]]

[24] G. J. Anders, A. Napieralski, and Z. Kulesza, "Calculation of the internal thermal resistance and ampacity of 3-core screened cables with fillers," IEEE Transactions on Power Delivery, vol. 14, no. 3, pp. 729-733, 1999.
[25] J.-A. Pérez-Rúa, K. Das, and N. A. Cutululis, "Optimum sizing of offshore wind farm export cables," International Journal of Electrical Power \& Energy Systems, vol. 113, no. December 2019, pp. 982-990, 2019. [Online]. Available: https://doi.org/10.1016/j.ijepes.2019.06.026

[26] J. J. Grainger and W. D. J. Stevenson, Power System Analysis, 2nd ed. McGraw-Hill Education, 1994.

[27] P. Sørensen, N. A. Cutululis, A. Vigueras-Rodríguez, L. E. Jensen, J. Hjerrild, M. H. Donovan, and H. Madsen, "Power Fluctuations From Large Wind Farms," IEEE Transactions on Power Systems, vol. 22, no. 3, pp. 958-965, 2007.

[28] S. Dutta and T. Overbye, "A Graph-theoretic Approach for Addressing Trenching Constraints in Wind Farm Collector System Design," in IEEE Power and Energy Conference at Illinois (PECI), 2013, pp. 1-5.

[29] A. Klein and D. Haugland, "Obstacle-aware optimization of offshore wind farm cable layouts," Annals of Operations Research, vol. 272, no. 1-2, pp. 373-388, 2017.

[30] A. C. Pillai, J. Chick, L. Johanning, M. Khorasanchi, and V. De Laleu, "Offshore wind farm electrical cable layout optimization," Engineering Optimization, vol. 47, no. 12, pp. 1689-1708, 2015.

[31] C. Berzan, K. Veeramachaneni, J. McDermott, and U.-M. O'Reilly, "Algorithms for cable network design on large-scale wind farms," Tech. Rep., 2011. [Online]. Available: https://thirld.com/files/msrp_techreport. pdf.[AccessedApril15,2019]

[32] IBM, "IBM ILOG CPLEX Optimization Studio CPLEX User Manual," Tech. Rep., 2015. [Online]. Available: https://www.ibm.com/support/ knowledgecenter.[AccessedApril11,2019]

[33] ESCA, SEAFISH, and RenewableUK, "KIS-ORCA." [Online]. Available: http://kis-orca.eu/about-us\#.XLRI_-gzYuU. [AccessedApril15,2019]

[34] ABB, "XLPE Submarine Cable Systems Attachment to XLPE Land Cable Systems - User's Guide," 2018. [Online]. Available: new.abb.com/docs/default-source/ewea... /xlpe-submarine-cable-systems-2gm5007.pdf.[AccessedOct29,2018]

[35] A. M. Jenkins, M. Scutariu, and K. S. Smith, "Offshore wind farm interarray cable layout," in IEEE PowerTech at Grenoble, 2013, pp. 1-6.

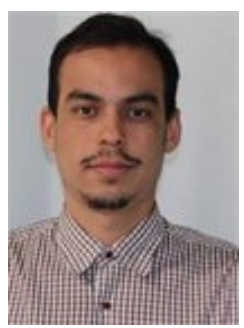

Juan-Andrés Pérez-Rúa received the B.Sc. degree in Electrical Engineering, with Summa Cum Laude distinction, from the Technological University of Bolivar, Colombia, in 2012, and the M.Sc. degree in Sustainable Transportation and Electrical Power Systems from the ISEC college, Coimbra, Portugal, the University of Nottinghan, England, and the University of Oviedo, Spain, in 2016. Currently, he is pursuing the Ph.D. degree in the Department of Wind Energy at the Technical University of Denmark (DTU). His present-day areas of interest include grid integration of renewables in power systems, and optimization.

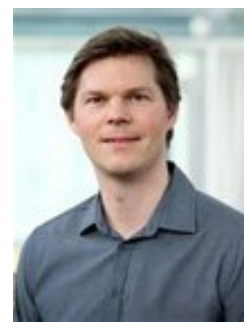

Mathias Stolpe received the Ph.D. degree in Optimization and Systems Theory from the Royal Institute of Technology (KTH), Stockholm, Sweden in 2003. He works as a Professor in the Department of Wind Energy at the Technical University of Denmark (DTU). His areas of research are structural and multidisciplinary optimization with focus on models and methods for global optimization.

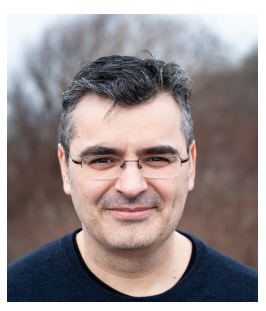

Nicolaos A. Cutululis received the M.Sc. and Ph.D. degrees, both in Automatic Control in 1998 and 2005, respectively. Currently, he is Professor in the Department of Wind Energy at the Technical University of Denmark. His main research interests are integration of wind power, with a special focus on offshore wind power, and grids. 